\title{
Résumé de la Déclaration sur la vaccination antigrippale pour la saison 2016-2017 du Comité consultatif national de l'immunisation (CCNI)
}

\author{
Gemmill I,2, Zhao $L^{3}$, Cochrane $L^{3}$ au nom du Comité consultatif national de l'immunisation \\ $(\mathrm{CCNI})^{4, *}$
}

\section{Résumé}

Contexte : La grippe est une infection respiratoire principalement causée par les virus de la grippe $A$ et $B$. La vaccination est le moyen le plus efficace de prévenir la grippe et ses complications. Le Comité consultatif national de l'immunisation (CCNI) fournit chaque année à l'Agence de la santé publique du Canada (l'Agence) des recommandations pour les vaccins antigrippaux saisonniers.

Objectif : Résumer les recommandations du CCNI concernant l'utilisation des vaccins contre la grippe saisonnière pour la saison grippale 2016-2017.

Méthodologie : Le Groupe de travail sur l'influenza du CCNI formule des recommandations sur la vaccination antigrippale annuelle et les soumet au CCNI aux fins d'examen et d'approbation, selon le processus d'élaboration des recommandations du CCNI fondé sur des données probantes. Ce processus comprend l'examen des points suivants : le fardeau de la maladie et les populations cibles, l'efficacité, l'immunogénicité et l'innocuité des vaccins antigrippaux, les calendriers de vaccination et d'autres aspects de l'immunisation contre la grippe. Ces recommandations sont publiées chaque année sur le site Web de l'Agence dans la déclaration du Comité consultatif national de l'immunisation : Chapitre sur la grippe du Guide canadien d'immunisation et Déclaration sur la vaccination antigrippale saisonnière (la Déclaration).

Résultats : Les recommandations annuelles du CCNI sur les vaccins contre la grippe saisonnière ont été mises à jour pour la saison grippale 2016-2017 et comprendront l'ajout des adultes souffrant de troubles neurologiques ou du développement neurologique parmi les groupes pour qui le vaccin antigrippal est particulièrement recommandé; comprendront le nouveau vaccin trivalent antigrippal inactivé à fortes doses pour les adultes âgés de 65 ans et plus; recommanderont que les personnes allergiques aux œufs puissent se faire vacciner contre la grippe au moyen du vaccin antigrippal vivant atténué (VAl) à faible teneur en ovalbumine homologué au Canada (le CCNI avait précédemment recommandé que les personnes allergiques aux œufs se fassent vacciner au moyen de vaccins antigrippaux trivalents inactivés); et retireront la recommandation préférentielle concernant l'utilisation du WVAl chez les enfants et les adolescents âgés de 2 à 17 ans. Deux addenda apportés à la déclaration de 2016-2017 portent sur ces nouvelles recommandations liées au WVAl.

Conclusion : Le CCNI continue de recommander la vaccination antigrippale annuelle pour toutes les personnes âgées de 6 mois et plus, et plus particulièrement pour les personnes présentant un risque élevé de complications ou d'hospitalisation liées à la grippe, les personnes susceptibles de transmettre la grippe à des sujets à risque élevé, ainsi que les autres personnes indiquées.

\author{
Affiliations \\ ${ }^{1}$ Président du Comité consultatif \\ national de l'immunisation \\ ${ }^{2}$ Bureau de santé de Kingston, \\ Frontenac, Lennox et Addington, \\ Kingston (Ontario) \\ ${ }^{3}$ Centre de l'immunisation \\ et des maladies respiratoires \\ infectieuses, Agence de la santé \\ publique du Canada, Ottawa \\ (Ontario) \\ ${ }^{4}$ Membres du CCNI fournis dans \\ les Remerciements
}

*Correspondance : naci-ccni@ phac-aspc.gc.ca

Citation proposée : Gemmill I, Zhao L, Cochrane L au nom du Comité consultatif national de l'immunisation (CCNI). Résumé de la Déclaration sur la vaccination antigrippale pour la saison 2016-2017 du Comité consultatif national de l'immunisation (CCNI). Relevé des maladies transmissibles au Canada 2016;42:210-4. https://doi.org/10.14745/ccdr.v42i09a06f

\section{Introduction}

La grippe fait partie des dix principales causes de décès au Canada (1). Bien que le fardeau de la grippe puisse varier d'une année à l'autre, il est estimé que dans une année donnée, il y a en moyenne 12200 hospitalisations (2) et environ 3500 décès attribuables à la grippe (3). Le Comité consultatif national de 
l'immunisation (CCNI) fournit chaque année à l'Agence de la santé publique du Canada (l'Agence) des recommandations pour les vaccins antigrippaux saisonniers. Les recommandations du CCNI sur l'utilisation du vaccin contre la grippe saisonnière pour la saison grippale 2016-2017 sont résumées ci-dessous. Tous les détails se trouvent dans la Déclaration sur la vaccination antigrippale pour 2016-2017 (4), qui comprend le chapitre du Guide canadien d'immunisation sur la grippe (section II de la Déclaration), et dans les deux addendas à la Déclaration $(5,6)$ publiés sur le site Web de l'Agence.

\section{Méthodologie}

Afin de préparer les recommandations relatives au vaccin contre la grippe saisonnière de 2016-2017, le Groupe de travail sur l'influenza (GTI) du CCNI a relevé et passé en revue les données probantes concernant les adultes atteints de troubles neurologiques ou du développement neurologique comme groupe à risque de développer des complications liées à la grippe; le nouveau vaccin antigrippal trivalent inactivé à fortes doses homologué au Canada pour les adultes âgés de 65 ans et plus (Fluzone ${ }^{\circledR}$ à forte dose [Sanofi Pasteur]); I'administration du vaccin antigrippal vivant atténué (VVAl) chez les personnes allergiques aux œufs; I'efficacité du vaccin (EV) du WVAl et du vaccin antigrippal inactivé. Après l'examen et I'analyse de ces renseignements, le GTI a proposé au CCNI des recommandations mises à jour relatives à l'utilisation des vaccins, selon le processus d'élaboration des recommandations du CCNI fondé sur des données probantes (7). Le CCNI a effectué l'évaluation critique des données probantes disponibles et il a approuvé les recommandations présentées. L'examen des données probantes concernant le vaccin Fluzone ${ }^{\circledR}$ à forte dose est publié séparément (8). La justification et les considérations pertinentes liées à toutes les recommandations mises à jour sont comprises dans la version intégrale de la déclaration de 2016-2017 (4) et les deux addendas à la déclaration de 2016-2017 sur I'utilisation du VVAI $(5,6)$.

\section{Résultats}

Nouveau pour la saison grippale 2016-2017

\section{Adultes atteints de troubles neurologiques ou du développement neurologique}

Depuis la saison 2015-2016, les enfants et les adolescents atteints de troubles neurologiques ou du développement neurologique (notamment troubles convulsifs, convulsions fébriles et retard de développement isolé) sont inclus dans le groupe présentant un risque élevé pour lequel la vaccination antigrippale est particulièrement recommandée. D'après l'examen préliminaire de la documentation et l'opinion d'experts, et conformément aux recommandations des autres pays, le CCNI inclut désormais les adultes atteints de troubles neurologiques ou du développement neurologique parmi les groupes à risque élevé pour lesquels le vaccin antigrippal est particulièrement recommandé. Par suite de l'examen préliminaire, on a noté que les cotes pour les complications de la grippe chez les patients atteints de maladies neurologiques par rapport aux patients n'en présentant pas allait de 1,57 (pneumonie : intervalle de confiance [IC] à $95 \%$, de 1,05 à 2,36 ) à 19,11 (admission en unité de soins intensifs : IC à $95 \%$, de 3,92 à 93,22) et 22,2 (hospitalisation : IC à $95 \%$ : de

2,6 à 186,0) (9-11). Dans les études examinées, parmi les pathologies considérées comme des facteurs de risque figuraient les maladies neuromusculaires, neurovasculaires, neurodégénératives ou neurodéveloppementales et les troubles convulsifs.

La justification et les conclusions de l'examen préliminaire de la documentation, ainsi que les recommandations mises à jour du CCNI pour l'ajout des adultes atteints de troubles neurologiques ou du développement neurologique sont publiés dans la déclaration de 2016-2017 (4).

\section{Nouveau vaccin trivalent antigrippal inactivé (Fluzone ${ }^{\circledR}$ à forte dose [Sanofi Pasteur])}

Le vaccin antigrippal Fluzone ${ }^{\circledR}$ à forte dose a été approuvé au Canada pour l'administration chez les adultes âgés de 65 ans et plus. Le vaccin Fluzone ${ }^{\circledR}$ à forte dose est un vaccin trivalent inactivé (VTI) contre la grippe contenant $60 \mu \mathrm{g}$ d'hémagglutinine (HA) par souche (comparativement à $15 \mu \mathrm{g}$ de HA par souche dans une dose normale), administré en dose de 0,5 mL par injection intramusculaire. D'après les données probantes disponibles, le CCNI conclut que le VTI à forte dose offre une protection supérieure par rapport à un VTI à dose normale pour les adultes âgés de 65 ans et plus. Cette protection relative supérieure par rapport à un VTI à dose normale semble augmenter avec l'âge chez les personnes de plus de 65 ans. Compte tenu du fardeau de la maladie associé à la grippe $\mathrm{A}(\mathrm{H} 3 \mathrm{~N} 2)$ et des données probantes à l'appui d'une efficacité supérieure du VTI à forte dose par rapport au VTI à dose normale, il semble que le VTI à forte dose conférerait la meilleure protection au groupe d'âge des personnes âgées de 65 ans et plus.

Un examen complet de la documentation sur le vaccin antigrippal Fluzone ${ }^{\circledR}$ à forte dose pour les adultes âgés de 65 ans et plus est publié séparément (8) et la justification entière du CCNI et les recommandations sur l'utilisation du vaccin sont publiés dans la déclaration 2016-2017 (4).

\section{Administration du VVAl aux personnes allergiques aux œufs}

L'innocuité de l'utilisation du WVAl chez les personnes allergiques aux œufs a été étudiée auprès de plus de 1100 enfants et adolescents (de 2 à 18 ans) au Royaume-Uni et au

Canada (12-14). Après un examen minutieux des études publiées récemment, le CCNI conclut que les personnes allergiques aux œufs peuvent être vaccinées contre la grippe en utilisant le VVAl à faible teneur en ovalbumine homologué au Canada. La dose complète de VVAl peut être utilisée sans test cutané au vaccin préalable et dans tous les milieux où les vaccins sont administrés régulièrement. Le VVAl semble également être bien toléré chez les personnes ayant des antécédents d'asthme stable ou de respiration sifflante récurrente; cependant, il reste contre-indiqué chez les sujets atteints d'asthme grave (c.-à-d. ceux qui reçoivent actuellement une glucocorticothérapie par voie orale ou de fortes doses de glucocorticoïdes par inhalation ou qui présentent une respiration sifflante active) ou ceux ayant une respiration sifflante qui a nécessité une intervention médicale au cours des sept jours précédant l'immunisation. 
L'examen de la documentation portant sur l'innocuité du WVAl chez les personnes allergiques aux œufs et la recommandation à jour du CCNI en matière d'administration du VVAl aux personnes allergiques aux œufs sont publiés dans un addenda à la déclaration de 2016-2017 (5).

Recommandations mises à jour du CCNI sur l'utilisation du VVAl chez les enfants âgés de 2 à 17 ans

Après un examen approfondi des études disponibles provenant des dernières saisons grippales, le CCNI conclut que les données probantes confirment que le WAI confère une protection contre la grippe comparable à celle que procure le vaccin antigrippal inactivé dans divers autres pays. Le Comité a d'ailleurs modifié ses recommandations concernant I'utilisation du vaccin contre la grippe chez les enfants âgés de 2 à 17 ans :

1. Chez les enfants qui ne présentent pas de contre-indications, les vaccins antigrippaux suivants peuvent être utilisés : VVAl quadrivalent, vaccin antigrippal quadrivalent inactivé (VAQ) ou VTI.

2. Les données probantes actuelles n'appuient pas une recommandation de l'utilisation préférentielle du VVAI chez les enfants et les adolescents âgés de 2 à 17 ans.

Compte tenu du fardeau du virus de la grippe B chez les enfants et du potentiel de non-concordance entre la souche prédominante du virus de la grippe $B$ en circulation et la souche d'un vaccin trivalent, le CCNI continue à recommander qu'un vaccin quadrivalent contre la grippe soit utilisé chez les enfants et les adolescents de 2 à 17 ans. En l'absence de VAQ, il faut utiliser le VTI.

Les données de l'étude d'observation examinées mettent en évidence le défi d'interpréter l'efficacité du WAI et du vaccin antigrippal inactivé lorsque les estimations ponctuelles par sous-type de grippe sont calculées en fonction d'échantillons de petite taille associés à de grands intervalles de confiance. Par conséquent, dans la formulation de cette recommandation, le CCNI reconnaît la nécessité de continuer à surveiller étroitement les données sur l'efficacité du WAI par sous-type de grippe et selon l'efficacité relative du WVAl par rapport à celle du vaccin antigrippal inactivé. Le CCNI a établi la nécessité de poursuivre les recherches afin de combler les lacunes actuelles en matière de connaissances :

3. Le CCNI encourage fortement que des recherches multidisciplinaires supplémentaires (p. ex., épidémiologiques, immunologiques, virologiques) soient menées pour établir les raisons qui expliquent les estimations discordantes de l'efficacité des vaccins de 2015-2016 entre les études et la faible efficacité du VVAI par rapport au virus de la grippe $\mathrm{A}(\mathrm{H} 1 \mathrm{~N} 1)$ pdm09 signalée dans certaines études.

4. Le CCNI recommande vivement que suffisamment de ressources soient consacrées à l'approfondissement des recherches sur la grippe et à l'amélioration des systèmes de surveillance sentinelle au Canada afin d'améliorer I'évaluation de l'efficacité potentielle et réelle des vaccins antigrippaux et de disposer des meilleures données probantes possibles pour les programmes de vaccination contre la grippe et les recommandations en la matière.
De plus amples détails et la justification à l'appui des recommandations mises à jour du CCNI sur I'utilisation du WAI chez les enfants âgés de 2 à 17 ans sont publiés dans un addenda à la déclaration de 2016-2017 (6).

Résumé des recommandations du CCNI concernant l'utilisation des vaccins contre la grippe pour la saison grippale 2016-2017

Le CCNI continue de recommander la vaccination antigrippale pour toutes les personnes âgées de 6 mois et plus, et plus particulièrement les personnes présentant un risque élevé de complications ou d'hospitalisation liées à la grippe, les personnes susceptibles de transmettre la grippe à des sujets à risque élevé, ainsi que les autres personnes indiquées au tableau 1.

\section{Tableau 1 : Groupes pour lesquels le vaccin antigrippal est particulièrement recommandé ${ }^{1}$}

Personnes présentant un risque élevé de complications liées à la grippe ou plus susceptibles de devoir être hospitalisées

- Toutes les femmes enceintes ${ }^{2}$.

- Adultes et enfants atteints d'une des affections chroniques suivantes :

maladies cardiaques ou pulmonaires (notamment dysplasie bronchopulmonaire, fibrose kystique et asthme);

diabète sucré ou autres maladies métaboliques:

cancer, troubles liés à l'immunodépression (résultant d'une maladie sous-jacente, d'un traitement, ou des deux);

néphropathie;

anémie ou hémoglobinopathie:

troubles neurologiques ou du développement neurologique ${ }^{3}$; obésité morbide (indice de masse corporelle [IMC] de 40 et plus); enfants et adolescents (âgés de 6 mois à 18 ans) sous traitement pendant de longues périodes par de l'acide acétylsalicylique, en raison de la possibilité d'un risque accru de syndrome de Reye associé à la grippe.

- Résidents de maisons de soins infirmiers et d'autres établissements de soins de longue durée, quel que soit leur âge.

- Personnes de 65 ans et plus.

- Tous les enfants âgés de 6 à 59 mois.

- Autochtones.

Personnes qui pourraient transmettre la grippe à des sujets à risque élevé

- Travailleurs de la santé et autres fournisseurs de soins dans des établissements et en milieux communautaires qui, par leurs activités, pourraient transmettre la grippe à des sujets à risque élevé de complications.

- Contacts familiaux (adultes et enfants) de personnes à risque élevé de complications liées à la grippe, que ces dernières aient été vaccinées ou non :

- contacts familiaux de personnes à risque élevé énumérées dans la section ci-dessus;

contacts familiaux des nourrissons de moins de 6 mois, qui sont à risque élevé de complications grippales, mais qui ne peuvent pas recevoir un vaccin contre la grippe;

membres d'un ménage devant accueillir un nouveau-né durant la saison grippale.

- Personnes qui ont régulièrement soin d'enfants âgés de 59 mois ou moins, que ce soit à la maison ou à l'extérieur.

- Personnes qui fournissent des services à des sujets à risque élevé dans un milieu fermé ou relativement fermé (p. ex. équipage de navire).

Autres

- Personnes qui fournissent des services communautaires essentiels.

- Personnes en contact direct avec de la volaille infectée par le virus de la grippe aviaire durant les activités d'abattage.

${ }^{1}$ Recommandations à jour en caractères gras

${ }^{2}$ Le risque d'hospitalisation liée à la grippe augmente avec la durée de gestation (c'est-à-dire qu'il est plus élevé pendant le troisième trimestre que pendant le deuxième)

${ }^{3}$ Sont inclus les troubles convulsifs, les convulsions fébriles et le retard de développement isolé chez les enfants, et les maladies neuromusculaires, neurovasculaires et neurodégénératives et neurodéveloppementales et les troubles convulsifs chez les adultes, mais sont exclues les migraines et les troubles neuropsychiatriques en l'absence d'une pathologie neurologique 
Les options de vaccination antigrippale recommandées par groupes d'âge et de risque particuliers et selon la posologie et la voie d'administration par âge sont résumées dans les tableaux 2 et $\mathbf{3}$, respectivement.

Tableau 2 : Choix du vaccin antigrippal pour certains groupes d'âge et certains groupes à risque (en l'absence de contre-indications)'

\begin{tabular}{|c|c|c|}
\hline $\begin{array}{l}\text { Groupes } \\
\text { d'âge }\end{array}$ & $\begin{array}{l}\text { Types de } \\
\text { vaccins } \\
\text { disponibles }\end{array}$ & Commentaires \\
\hline $\begin{array}{l}\text { Enfants de } \\
6 \text { à } 23 \text { mois }\end{array}$ & $\begin{array}{ll}\text { - } & \text { VTI } \\
\text { - } & \text { VAQ } \\
\text { VTla }\end{array}$ & $\begin{array}{l}\text { Le VTI, le VAQ et le VTla sont autorisés pour ce } \\
\text { groupe d'âge. } \\
\text { Le CCNI recommande, compte tenu du fardeau } \\
\text { présenté par la grippe de type B, d'utiliser le } \\
\text { VAQ. Si le VAQ n'est pas offert, le VTI, avec ou } \\
\text { sans adjuvant, doit être utilisé. }\end{array}$ \\
\hline $\begin{array}{l}\text { Enfants de } \\
2 \text { à } 17 \text { ans }\end{array}$ & $\begin{array}{ll}\text { - } & \text { VTI } \\
\text { - } & \text { VAQ } \\
& \text { quadrivalent }\end{array}$ & $\begin{array}{l}\text { Chez les enfants qui ne présentent pas de } \\
\text { contre-indications, les vaccins antigrippaux } \\
\text { suivants peuvent être utilisés : VVAI, VQI ou } \\
\text { VTI. } \\
\text { Les données probantes actuelles n'appuient } \\
\text { pas une recommandation de I'utilisation } \\
\text { préférentielle du VVAI chez les enfants et les } \\
\text { adolescents âgés de } 2 \text { à } 17 \text { ans. } \\
\text { Compte tenu du fardeau du virus de la } \\
\text { grippe B chez les enfants et du potentiel } \\
\text { de non-concordance entre la souche } \\
\text { prédominante du virus de la grippe B en } \\
\text { circulation et la souche d'un vaccin trivalent, le } \\
\text { CCNI continue à recommander qu'un vaccin } \\
\text { quadrivalent contre la grippe soit utilisé chez } \\
\text { les enfants et les adolescents de } 2 \text { à } 17 \text { ans. En } \\
\text { I'absence de VAQ, il faut utiliser le VTI. } \\
\text { II n'est pas recommandé d'administrer le VVAI } \\
\text { aux enfants immunodéprimés. } \\
\text { Le VVAI, le VTI ou le VAQ peuvent être } \\
\text { utilisés chez les enfants atteints d'affections } \\
\text { chroniques, y compris I'asthme non grave }{ }^{2} \text { et la } \\
\text { fibrose kystique sans immunosuppression. }\end{array}$ \\
\hline $\begin{array}{l}\text { Adultes de } \\
18 \text { à } 59 \text { ans }\end{array}$ & $\begin{array}{ll}\text { - } & \text { VTI } \\
\text { - } & \text { VAQ } \\
\text { VAI } & \text { quadrivalent }\end{array}$ & $\begin{array}{l}\text { Le VTI et le VAQ sont les vaccins recommandés } \\
\text { pour les adultes atteints d'affections } \\
\text { chroniques. } \\
\text { Le VTI et le VAQ sont recommendés pour les } \\
\text { travailleurs de la santé. } \\
\text { L'administration du VVAI n'est pas } \\
\text { recommandée chez les adultes } \\
\text { immunodéprimés. }\end{array}$ \\
\hline $\begin{array}{l}\text { Adultes de } \\
60 \text { à } 64 \text { ans }\end{array}$ & $\begin{array}{ll}\text { - } & \text { VTI } \\
\text { - VAQ }\end{array}$ & $\begin{array}{l}\text { Le VTI et le VAQ sont autorisés pour ce groupe } \\
\text { d'âge. }\end{array}$ \\
\hline $\begin{array}{l}\text { Adultes } \\
\text { âgés de } \\
65 \text { ans et } \\
\text { plus }\end{array}$ & $\begin{array}{ll}\text { - } & \text { VTI } \\
\text { - } & \text { VAQ } \\
\text { - } & \text { VTla } \\
\text { - } & \text { VTI à forte } \\
& \text { dose }\end{array}$ & $\begin{array}{l}\text { Compte tenu du fardeau de la } \\
\text { grippe } \mathrm{A}(\mathrm{H} 3 \mathrm{~N} 2) \text { et des preuves indiquant } \\
\text { une plus grande efficacité pour ce groupe } \\
\text { d'âge, un VTI à forte dose devrait conférer } \\
\text { une meilleure protection chez les personnes } \\
\text { âgées que le vaccin intramusculaire à dose } \\
\text { normale. }\end{array}$ \\
\hline $\begin{array}{l}\text { Femmes } \\
\text { enceintes }\end{array}$ & $\begin{array}{ll}\text { - VTI } \\
\text { - VAQ }\end{array}$ & $\begin{array}{l}\text { L'administration du WVAl n'est pas } \\
\text { recommandée en raison du risque théorique } \\
\text { que ferait encourir au fœtus l'administration } \\
\text { d'un vaccin à virus vivants. }\end{array}$ \\
\hline
\end{tabular}

Abréviations : VTla, vaccin antigrippal trivalent inactivé avec adjuvant; VAI, vaccin antigrippal vivant atténué (préparation quadrivalente); $\mathrm{VAQ}$, vaccin antigrippal quadrivalent inactivé; VTI, vaccin antigrippal trivalent inactivé

Recommandations à jour en caractères gras

${ }^{2}$ Une personne présentant de l'asthme grave est définie comme étant une personne qui prend des glucocorticoïdes par inhalation, par voie orale ou en fortes doses, présentant un sifflement actif, ou ayant souffert d'une respiration sifflante ayant nécessité une intervention médicale au cours des sept jours précédant la vaccination
Tableau 3 : Posologie et voie d'administration recommandées pour le vaccin antigrippal, selon l'âge, pour la saison 2016-2017

\begin{tabular}{|c|c|c|c|c|}
\hline $\begin{array}{l}\text { Groupe } \\
\text { d'âge }\end{array}$ & $\begin{array}{c}\text { VTI sans } \\
\text { adjuvant } \\
\text { ou VAQ IM } 1\end{array}$ & $\begin{array}{c}\text { VTI contenant } \\
\text { l'adjuvant MF59 } \\
\text { (Fluad } \\
\text { Pédiatrique } \\
\text { Fluad ou }{ }^{\mathrm{MC}} \text { IM }\end{array}$ & $\begin{array}{l}\text { VVAI (FluMist@ } \\
\text { Quadrivalent) IN }\end{array}$ & $\begin{array}{l}\text { Nombre } \\
\text { de doses } \\
\text { requises }\end{array}$ \\
\hline 6 à 23 mois & $0,5 \mathrm{~mL}^{2}$ & $0,25 \mathrm{~mL}$ & - & 1 ou $2^{3}$ \\
\hline 2 à 8 ans & $0,5 \mathrm{~mL}$ & - & $\begin{array}{r}0,2 \mathrm{~mL}(0,1 \mathrm{~mL} \\
\text { par narine })\end{array}$ & 1 ou $2^{3}$ \\
\hline 9 à 17 ans & $0,5 \mathrm{~mL}$ & - & $\begin{array}{r}0,2 \mathrm{~mL}(0,1 \mathrm{~mL} \\
\text { par narine })\end{array}$ & 1 \\
\hline 18 à 59 ans & $0,5 \mathrm{~mL}$ & - & $\begin{array}{r}0,2 \mathrm{~mL}(0,1 \mathrm{~mL} \\
\text { par narine })\end{array}$ & 1 \\
\hline 60 à 64 ans & $0,5 \mathrm{~mL}$ & - & - & 1 \\
\hline$\geq 65$ ans & $0,5 \mathrm{~mL}$ & $0,5 \mathrm{~mL}$ & - & 1 \\
\hline
\end{tabular}

Abréviations : IM, intramusculaire; IN, intranasal; WVI, vaccin antigrippal vivant atténué (préparation quadrivalente); VAQ, vaccin antigrippal quadrivalent inactivé; VTI, vaccin antigrippal trivalent inactivé

Influvac ${ }^{\circledast} 18$ ans et plus, Fluviral ${ }^{\circledast} 6$ mois et plus, Agriflu ${ }^{\circledast} 6$ mois et plus, Vaxigrip ${ }^{\circledR} 6$ mois et plus, Fluzone ${ }^{\circledast} 6$ mois et plus, Fluzone ${ }^{\circledast}$ à forte dose 65 ans et plus, Flulaval ${ }^{\circledR}$ Tetra 6 mois et plus, et Fluzone ${ }^{\circledR}$ quadrivalent 6 mois et plus

${ }^{2}$ Ces renseignements diffèrent de ceux contenus dans la monographie de produit

${ }^{3}$ Les enfants âgés d'au moins 6 mois et de moins de 9 ans qui ne se sont jamais fait vacciner contre la grippe saisonnière doivent recevoir deux doses du vaccin à au moins quatre semaines d'intervalle. Les enfants admissibles de moins de 9 ans qui ont déjà reçu une dose ou plus du vaccin antigrippal saisonnier dans le passé devraient recevoir une dose par saison, au cours des années suivantes

\section{Conclusion}

Le CCNI continue de recommander la vaccination antigrippale annuelle pour toutes les personnes âgées de 6 mois et plus (en tenant compte des indications et contre-indications selon l'âge propres à chaque produit), et plus particulièrement les personnes présentant un risque élevé de complications ou d'hospitalisation liées à la grippe, notamment toutes les femmes enceintes, les personnes susceptibles de transmettre la grippe à des sujets à risque élevé, ainsi que les autres personnes indiquées.

\section{Remerciements}

Membres du CCNI : I. Gemmill (président), C. Quach-Thanh (vice-présidente), N. Dayneka, S. Deeks, B. Henry, S. Marchant-Short, M. Salvadori, N. Sicard, W. Vaudry, D. Vinh, R. Warrington

\section{Anciens membres du CCNI : D. Kumar}

Agents de liaison : J. Blake (Société des obstétriciens et gynécologues du Canada), J. Brophy (Association canadienne pour la recherche et l'évaluation en immunisation), A. Cohn (Centers for Disease Control and Prevention, États-Unis [CDC]), J. Emili (Collège des médecins de famille du Canada), M. Lavoie (Conseil des médecins hygiénistes en chef), C. Mah (Association canadienne de santé publique), D. Moore (Société canadienne 
de pédiatrie), A. Pham-Huy (Association pour la microbiologie médicale et l'infectiologie Canada), E. Sartison (Comité canadien d'immunisation [CCl])

Anciens agents de la liaison : C. Weinbaum (CDC)

Représentants d'office : K. Barnes (Défense nationale et Forces armées canadiennes), G. Charos (Centre de l'immunisation et des maladies respiratoires infectieuses [CIMRI], Agence de la santé publique du Canada [ASPC] et CCI), G. Coleman (Direction des produits biologiques et des thérapies génétiques, Santé Canada), J. Gallivan (Direction des produits de santé commercialisés, Santé Canada), J. Pennock (CIMRI, ASPC), T. Wong (Direction générale de la santé des Premières nations et des Inuits, Santé Canada)

Le CCNI tient également à souligner la contribution de L. Grohskopf (CDC), J. Langley (groupe de travail sur l'influenza [GTI]), J. McElhaney (GTI), A. McGeer (GTI), V. Meikle (CIMRI, ASPC), R. Stirling (CIMRI, ASPC), B. Warshawsky (GTI), E. Wong (CIMRI, ASPC) et J. Xiong (GTI)

\section{Financement}

Les travaux du CCNI sont appuyés par l'Agence de la santé publique du Canada.

\section{Conflit d'intérêts}

Aucun.

\section{Références}

1. Statistique Canada. Les dix principales causes de décès, 2011. Ottawa: Statistique Canada; 2014 [Date de modification : le 27 nov 2015; cité le 16 août 2016]. http:// www.statcan.gc.ca/pub/82-625-x/2014001/article/11896-fra. htm.

2. Schanzer DL, McGeer A, Morris K. Statistical estimates of respiratory admissions attributable to seasonal and pandemic influenza for Canada. Influenza Other Respir Viruses 2013;7(5):799-808.

3. Schanzer DL, Sevenhuysen C, Winchester B, Mersereau T. Estimating influenza deaths in Canada, 1992-2009. PLoS One 2013;8(11):e80481.

4. Comité consultatif national de l'immunisation (CCNI). Déclaration sur la vaccination antigrippale pour la saison 2016-2017. Ottawa: PHAC; 2016 [Date de modification : le 29 mai 2016; cité le 16 août 2016]. http://www.phac-aspc. gc.ca/naci-ccni/flu-2016-grippe-fra.php.
5. Comité consultatif national de l'immunisation (CCNI). ADDENDA - I'administration du VVAI aux personnes allergiques aux œufs. [Internet]. Ottawa : ASPC; 2016 [Date de modification le 31 août 2016]. http://www.phac-aspc. gc.ca/naci-ccni/flu-2016-grippe-addendum-eggs-oeufs-fra. php.

6. Comité consultatif national de l'immunisation (CCNI). ADDENDA - Utilisation du WVAl chez les enfants et les adolescents. [Internet]. Ottawa : ASPC 2016 [Date de modification le 41 août 2016]. http://www.phac-aspc.gc.ca/ naci-ccni/flu-2016-grippe-addendum-children-enfants-fra. php.

7. Comité consultatif national de l'immunisation (CCNI). Recommandations pour l'immunisation fondées sur des données probantes - Méthodes du Comité consultatif national de l'immunisation. Relevé des maladies transmissibles au Canada 2009;35:ACS-1. http://www.phacaspc.gc.ca/publicat/ccdr-rmtc/09vol35/acs-1/index-fra.php.

8. Comité consultatif national de l'immunisation (CCNI). Examen de la littérature sur le vaccin contre la grippe saisonnière à forte dose chez les adultes de 65 ans et plus. Ottawa: PHAC; 2016 [Date de modification : le 11 avr 2016; cité le 16 août 2016]. http://www.phac-aspc.gc.ca/naci-ccni/ influenza-vaccine-65-plus-vaccin-contre-la-grippe-65-plus-fra. php.

9. Gilca R, De Serres G, Boulianne N, Ouhoummane N, Papenburg J, Douville-Fradet M, et al. Risk factors for hospitalization and severe outcomes of 2009 pandemic H1N1 influenza in Quebec, Canada. Influenza Other Respir Viruses 2011;5(4):247-55.

10. Mertz D, Kim TH, Johnstone J, Lam PP, Science M, Kuster $\mathrm{SP}$, et al. Populations at risk for severe or complicated influenza illness: systematic review and meta-analysis. BMJ 2013;347:f5061.

11. Poeppl W, Hell M, Herkner H, Stoiser B, Fritsche G, SchurzBamieh N, et al. Clinical aspects of 2009 pandemic influenza A (H1N1) virus infection in Austria. Infection 2011;39(4):34152.

12. Turner PJ, Southern J, Andrews NJ, Miller E, ErlewynLajeunesse M; SNIFFLE Study Investigators. Safety of live attenuated influenza vaccine in atopic children with egg allergy. J Allergy Clin Immunol 2015;136(2):376-81.

13. Turner PJ, Southern J, Andrews NJ, Miller E, ErlewynLajeunesse M; SNIFFLE-2 Study Investigators. Safety of live attenuated influenza vaccine in young people with egg allergy: multicenter prospective cohort study. BMJ 2015;351:h6291.

14. Des Roches A, Samaan K, Graham F, et al. Safe vaccination of patients with egg allergy by using live attenuated influenza vaccine. J Allergy Clin Immunol Pract 2015;3(1):138-9. 